\title{
ALCANCE
}

\section{AVALIAÇÃO DA NECESSIDADE DE TREINAMENTO AMBIENTAL: UM ESTUDO EM TRÊS EMPRESAS DO SETOR QUÍMICO BRASILEIRO}

ENVIRONMENTAL TRAINING NEEDS ASSESSMENT: A STUDY OF THREE COMPANIES IN THE BRAZILIAN CHEMICAL SECTOR

EVALUACIÓN DE LA NECESIDAD DE ENTRENAMIENTO AMBIENTAL: UN ESTUDIO EN TRES EMPRESAS DEL SECTOR QUÍMICO BRASILEÑO

MARCO ANTONIO BATISTA DA SILVA

Doutor

Universidade Nove de Julho - Brasil

ORCID: 0000-0003-4616-4038

prof.marcoabs@gmail.com

PRISCILA REZENDE DA COSTA

Doutora

Universidade Nove de Julho - Brasil

ORCID: 0000-0002-7012-0679

priscilarezende@yahoo.com.br

CLAUDIA TEREZINHA KNIESS

Doutora

Universidade Nove de Julho - Brasil

ORCID: 0000-0002-1961-2037

kniesscl@yahoo.com.br

Submetido em: 20/06/2018

Aprovado em: 03/06/2019

INDEXED BY

Doi: 10.14210.alcance.v26n3(set/dez).p382-398

"redalyc.org

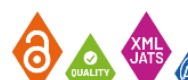

()

\section{.}

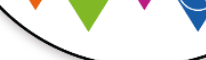

\section{LCENÇA CC BY:}

Artigo distribuído

sob os termos

Creative Commons,

permite uso e

distribuição irrestrita

em qualquer meio

desde que o autor

credite a fonte

original.

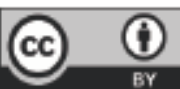




\section{RESUMO}

Neste artigo o objetivo foi analisar o alinhamento do processo de Avaliação da Necessidade de Treinamento (ANT) ambiental em três grandes empresas do setor químico brasileiro que tem a sustentabilidade como fator estratégico de vantagem competitiva. Tal estudo justifica-se pela necessidade de se aprofundar estudos sobre o papel da ANT como tema que ainda apresenta poucos estudos alinhados aos objetivos e ás estratégias organizacionais, visando à dimensão ambiental da sustentabilidade no contexto do setor químico brasileiro. Utilizou-se a abordagem de pesquisa qualitativa, com estudo de casos múltiplos baseado em triangulação de fontes de evidências. Os resultados confirmam que a sistematização da avaliação da necessidade de treinamento ambiental, por meio de sua abrangência individual, grupal e organizacional e da utilização da abordagem proativa pode potencializar o desenvolvimento de competências individuais essenciais à sustentabilidade a partir dos gaps de competências identificados. As contribuições gerenciais relacionam-se à descrição da fase de ANT desenvolvida pelas empresas estudadas, podendo servir de benchmark para outras empresas do setor, bem como apresentar às empresas estudadas possibilidade de aprimoramento de seu processo. No contexto acadêmico, busca contribuir para a investigação da abrangência das abordagens do processo de avaliação da necessidade de treinamento ambiental, aprofundando a investigação sobre os temas.

Palavras-chave: Avaliação da Necessidade de Treinamento, Treinamento Ambiental, Indústrias Químicas.

\section{ABSTRACT}

The aim of this article was to analyze the alignment of Environmental Training Needs Assessment (TNA) process in three large companies in the Brazilian chemical sector, for which sustainability is a strategic factor of competitive advantage. This study is justified by the need to deepen studies on the role of TNA, as a theme on which there are few studies aligned with the organizational goals and strategies, focusing on the environmental dimension of sustainability in the context of the Brazilian chemical sector. We used the qualitative research approach, with multiple case studies based on triangulation of sources of evidence. The results confirm that the systematization of environmental training needs assessment, through its individual, group and organizational comprehensiveness and the use of proactive approaches, can potentiate the development of individual competences essential to sustainability, based on the skills gaps identified. The managerial contributions are related to the description of the TNA phase developed by the companies studied, which can serve as a benchmark for other companies in the sector. It also presents the companies studied with an opportunity to improve their process. In the academic context, it is hoped that this work will contribute to research on the comprehensiveness of the approaches to environmental training needs assessment, furthering research on these themes.

Key words: Training Needs Assessment, Environmental Training, Chemical Industry.

\section{RESUMEN}

En este artículo el objetivo fue analizar la alineación del proceso de Evaluación de la Necesidad de Entrenamiento (ANT) ambiental en tres grandes empresas del sector químico brasileño que tiene la sustentabilidad como factor estratégico de ventaja competitiva. Tal estudio se justifica por la necesidad de aprofundar estudios sobre el papel de la ANT como tema que todavia presenta pocos estudios alineados a los objetivos y a las estratégias organizacionales, visando a la dimensión ambiental de la sustentabilidad en el contexto del sector químico brasileño. Se utilizó el abordaje de investigación cualitativa, con estudio de casos múltiples baseado en triangulación de fuentes de evidencias. Los resultados confirman que la sistematización de la evaluación de la necesidad de entrenamiento ambiental, por medio de su alcance individual, grupal y organizacional y de la utilización del abordaje proativo puede potencializar el desarrollo de competencias individuales esenciales a la sustentabilidad a partir de los gaps de competencias identificadas. Las contribuciones gerenciales se relacionan a la descripción de la fase de ANT desarrollada por las empresas estudiadas, pudiendo servir de benchmark para otras empresas del sector, como también presentar a las empresas estudiadas posibilidad de mejora de su proceso. En el contexto académico, busca contribuir para la investigación del alcance de los abordajes del proceso de evaluación de la necesidad de entrenamiento ambiental, aprofundando la investigación sobre los temas.

Palabras-clave: Evaluación de la Necesidad de Entrenamiento, Entrenamiento Ambiental, Industrias Químicas. 


\section{INTRODUÇÃO}

O tema sustentabilidade tem apresentado um interesse crescente pela sociedade, pelos governos e pelas organizações. A indústria química brasileira, que ocupa o terceiro lugar no desenvolvimento do Produto Interno Bruto (PIB) industrial brasileiro e, em 2015, a oitava colocação em termos de faturamento líquido do mundo (Abiqum, 2017), tem apresentado destaque no tema sustentabilidade (Prado, 2003; Ferreira, Roch, \& Silva, 2013; Abiquim, 2017). Soto (2012) afirma que a adoção da sustentabilidade pelas indústrias químicas pode ser uma grande oportunidade para 0 desenvolvimento do setor. Estudos afirmam que a preocupação com o desenvolvimento de estratégias, políticas e práticas que busquem estimular os aspectos econômico, social e ambiental integrados à estratégia de sustentabilidade organizacional deve estar presente no desenvolvimento da gestão organizacional, com destaque para a gestão de pessoas (Collins, Lawrence, Pavlovich, \& Ryan, 2007; Jabbour \& Santos, 2008).

Diante desse contexto, o treinamento ambiental apresenta-se com papel importante para as empresas que "abraçam a filosofia da sustentabilidade" (Jackson, Schuler, \& Jiang, 2014, p. 40; Jabbour \& Santos, 2008), integrando os processos de treinamento à estratégia organizacional (Leite et al., 2009). Jabbour (2013) destaca a necessidade de trabalhos que tenham como foco o estudo das fases do processo de treinamento ambiental, buscando contribuir para 0 avanço no conhecimento no que tange ao tema treinamento ambiental. Abbad e Mourão (2012) identificaram que pouco se progrediu na última década sobre avaliação das necessidades de treinamento. Este autores constataram que o tema ainda se apresenta pouco alinhado aos objetivos e às estratégias organizacionais, sendo que ainda há maior produção no nível das tarefas e pouco estudo no nível organizacional, tanto na literatura nacional quanto internacional.

Diante dessa problemática, propõe-se a seguinte questão de pesquisa: como se dá o alinhamento do processo de avaliação do treinamento ambiental com o desenvolvimento de competências para sustentabilidade em três grandes empresas do setor químico brasileiro?

A partir do contexto apresentado no que tange à importância da sustentabilidade para as indústrías químicas e a necessidade de desenvolvimento de estudos no que diz respeito ao processo de avaliação da necessidade de treinamento ambiental, enuncia-se como objetivo geral deste trabalho analisar o alinhamento do processo de avaliação da necessidade de treinamento ambiental em três grandes empresas do setor químico brasileiro que tem a sustentabilidade como fator estratégico de vantagem competitiva. Este trabalho está estruturado em seis partes, sendo elas: a introdução; o referencial teórico; os aspectos metodológicos da pesquisa; a apresentação e discussão dos resultados; as considerações finais e, por fim, as referências.

\section{REFERENCIAL TEÓRICO}

Diversos pesquisadores têm desenvolvido estudos sobre treinamento ambiental, buscando entender e construir conhecimentos sobre esse campo de estudo. Dois trabalhos de revisão da literatura sobre o tema foram identificados: Jabbour (2013) e Moraes e Battistelle (2015), que destacam: o papel do treinamento ambiental para o desenvolvimento de inovações em PMEs; 0 treinamento ambiental como única prática de gestão de pessoas integrada ao sistema de gestão ISO 14001; o modelo de integração do treinamento ambiental aos níveis estratégico, tático e operacional; as diferentes intensidades de treinamento ambiental sendo oferecidas em diferentes empresas; o treinamento ambiental sendo apresentado como oportunidade de inclusão do tema sustentabilidade nos estudos da área de gestão de pessoas; a complexidade dos fatores que influenciam o treinamento ambiental e a apresentação da necessidade de novos estudos; 0 efeito positivo do treinamento ambiental dos funcionários sobre o desempenho da empresa no desenvolvimento sustentável; o papel do treinamento ambiental no alinhamento das práticas de gestão de pessoas à dimensão da sustentabilidade ambiental; a importância do treinamento ambiental e a consciência ambiental dos profissionais de gestão de pessoas (Baccaro et al., 2015); a correlação positiva entre o treinamento ambiental e as práticas da cadeia de suprimentos (Teixeira et al., 2016).

As práticas de gestão de pessoas têm um papel importante em buscar o envolvimento dos trabalhadores no controle da gestão ambiental, sendo o treinamento um fator de sucesso para a gestão ambiental (Jabbour, 2011; 2015), fornecendo ferramentas, técnicas e métodos que auxiliem no desenvolvimento de estratégias de gestão como alternativas para minimizar os impactos ambientais, sociais e econômicos, incluindo os interesses das comunidades locais; e possibilitando a aplicação de tais ferramentas de gestão (Unnikrishann, \& Hedge, 2007). Surge, assim, a necessidade de se desenvolver programas de treinamento ambiental especializado e customizado para se adequar às necessidades da organização (Cherian \& Jacob, 2012), que podem se adequar à conformidade com a regulamentação, definições de responsabilidades e deficiências dos empregados, desenvolver uma imagem positiva ou estimular a motivação dos empregados para participarem de forma proativa na gestão ambiental (Daily \& Huang, 2001). 
Tomando-se por base os diversos conceitos, o treinamento ambienal pode ser definido como um processo de treinamento no trabalho e de educação continuada que busca atingir os objetivos e os propósitos da gestão ambiental corporativa, habiliando todos os níveis organizacionais a integrar o desempenho da organização às questões ambientais (Teixeira et al., 2016). A escolha de tal definição justifica-se pelo fato de o conceito destacar a dimensão estratégica do treinamento ambiental, pois não é caracterizado somente como uma dimensão técnica, abrangendo aqueles que atuam em cargos da gestão ambiental, mas se estende a todos os níveis organizacionais. Também não está associado somente a uma ação instrucional, mas a um processo contínuo de educação, tendo como objetivo o desempenho organizacional na dimensão ambiental.

O treinamento ambiental, caracterizado como um processo, alinha-se a três subprocessos: avaliação da necessidade de treinamento; desenvolvimento de estratégias de treinamento e avaliação de treinamento, estabelecidos por Dermol e Carter (2013). Considerando-se o foco deste estudo, a seguir desenvolve-se a fundamentação teórica sobre avaliação da necessidade de treinamento.

Reed e Vakola (2006), Ferrreira e Abbad (2013) consideraram a avaliação de necessidade de treinamento como uma estratégia para ampliar o foco do treinamento e do desenvolvimento na organização. No entanto, os autores afirmaram que há pouco estudo que busca determinar a formação adequada ao desenvolvimento de oportunidades de aprendizagem, necessárias para se atingir os objetivos da organização e que a área de análise das necessidades de treinamento possui lacunas a serem preenchidas. Tais estudos corroboram os achados de Salas e Cannon-Bowers (2001) que, ao analisarem uma década dos estudos sobre treinamento, ressaltaram que há pouco trabalho empírico sobre análise da necessidade de treinamento, desenvolvimento e educação - TD\&E.

Abbad e Mourão (2012, p. 107) afirmaram que "avaliações de necessidades objetivam, pois, diagnosticar ou prognosticar necessidades de treinamento, de modo que elas, transformadas em objetivos educacionais, facilitem 0 desempenho de situações de aprendizagem para desenvolvê-las". Dessa forma, a análise das necessidades de treinamento abrange tanto as descrições de lacunas de conhecimento, habilidades e atitudes, que são componentes básicos das competências, voltadas para o trabalho, quanto as lacunas nas competências relacionadas às diversas esperas requeridas pela sociedade. Sendo assim, "esse processo se inicia com um diagnóstico adequado das necessidades de treinamento" (Abbad \& Mourão, 2012, p. 110).

Narashimhan e Ramanarayanan (2014) afirmaram que a análise do gap entre as competências que as pessoas na organização possuem e aquelas necessárias para se alcançarem os objetivos organizacionais deve ser a rota a ser seguida pela análise das necessidades de treinamento, considerando três níveis de análise: organizacional, envolvendo as necessidades oriundas da missão e das estratégias de organização; operacional, que analisa as exigências das competências que contribuem para o alcance dos objetivos de cada trabalho; e nível individual que avalia o gap do desempenho de cada indivíduo de acordo com padrões estabelecidos.

Salas e Cannon-Bowers (2001) afirmaram que a análise das necessidades é focada no processo de decidir quem e o que deve ser treinado. Seu foco deve ser determinar onde o treinamento é necessário, o que necessita ser ensinado e quem precisa ser treinado, de forma a focar na congruência entre os objetivos do treinamento, as metas organizacionais, os recursos disponíveis, as restrições e os suportes à transferência do aprendizado.

Abbad e Mourão (2012, p. 109) corroboraram a definição de Salas e Cannon-Bowers (2001), ao afirmarem que "uma avaliação da necessidade além da descrição de lacunas em Conhecimento, Habilidades e Atitudes (CHAs), componentes básicos das competências, objetiva a descrição do perfil do público-alvo e de seus contextos para o fim de desenvolvimento de estratégias instrucionais".

Ferreira e Abbad (2013) realizaram um levantamento das publicações científicas sobre treinamento, buscando identificar os estudos que tratavam de análise da necessidade de treinamento e identificaram como pertinentes aos objetivos da pesquisa 51 artigos publicados entre 1990 e 2010. Os autores identificaram um considerável avanço nos estudos sobre o tema, no entanto constataram que ainda há alguns gaps nos campos metodológico e teórico, indicando como poderiam ser ampliados os estudos sobre o tema, conforme Figura 1. 


\begin{tabular}{|c|c|c|}
\hline Dimensão & Como está & Como poderia ser \\
\hline Foco & $\begin{array}{l}\text { Diagnóstico/processual } \\
\text { Reativo }\end{array}$ & Proativo \\
\hline Teórica & $\begin{array}{l}\text { Pesquisas principalmente aplicadas com } \\
\text { investigação de solução para problemas práticos e } \\
\text { metodológicos }\end{array}$ & $\begin{array}{l}\text { Buscar desenvolvimento teórico e/ou de avaliação. } \\
\text { Uso da literatura de competência e gestão de } \\
\text { competências }\end{array}$ \\
\hline Metodológica & $\begin{array}{l}\text { Prevalecem estudos do tipo survey. } \\
\text { Relativa predominância da abordagem } \\
\text { quantitativa. } \\
\text { Relativa predominância do uso de questionário } \\
\text { como instrumento de coleta de dados. }\end{array}$ & $\begin{array}{l}\text { Uso de múltiplos métodos de coleta e de análise de dados } \\
\text { como nas ciências sociais e comportamentais. } \\
\text { Desenvolvimento de estudos com abordagens qualitativa } \\
\text { e quantitativa simultaneamente. } \\
\text { Utilização de amostras mais heterogêneas com } \\
\text { empregados de diferentes níveis. }\end{array}$ \\
\hline $\begin{array}{l}\text { Nível } \\
\text { análise }\end{array}$ & $\begin{array}{l}\text { Relativa predominância em estudos no nível micro, } \\
\text { ou seja, do indivíduo ( } 16 \text { estudos), nível mesmo ( } 8 \\
\text { estudos) e nível macro ( } 8 \text { estudos). }\end{array}$ & $\begin{array}{l}\text { Adoção de modelos multiníveis: organizacional (contexto } \\
\text { interno e externo); área ou unidade organizacional; e } \\
\text { individual. }\end{array}$ \\
\hline
\end{tabular}

Figura 1. Panorama dos estudos sobre Análise das Necessidades de Treinamento

Fonte: Elaborada pelo autor adaptado de Ferreira e Abbad (2013)

Arshad, Yusof, Mahmood, Ahmed e Akthar (2015), embasados em autores seminais como Anderson (1994), Berger (1993), Wright e Geroy (1992), descreveram as duas abordagens presentes no desenvolvimento da avaliação das necessidades de treinamento: a abordagem reativa e a proativa. Em uma perspectiva reativa, a avaliação das necessidades pauta-se na diferença entre o desempenho no trabalho esperado e realizado, não tendo como foco a ligação entre as competências necessárias aos objetivos estratégicos da organização. $\mathrm{Na}$ abordagem proativa, há a ampliação da área de análise, buscando-se avaliar o ambiente de negócios em que a alta gestão é inserida na avaliação, visando à determinação de competências necessárias às mudanças importantes que ocorrem na organização, a fim de responder às exigências fundamentais para a competitividade dessa organização.

Salas e Cannon-Bowers (2001) propuseram um modelo de análise das necessidades, destacando três categorias gerais: a) o que já é trazido pelos treinados; b) as variáveis que engajam o treinado no processo de participar do treinamento e aprender; e c) como o treinamento pode maximizar a experiência de aprendizagem. A Figura 2 sintetiza tais categorias que, considerando o nível organizacional, demonstram a influência que o ambiente organizacional pode ter na aplicação no trabalho dos novos conhecimentos, habilidades e atitudes adquiridos, entendendo, dessa forma, não somente os conhecimentos e as habilidades necessários, mas também os fatores que permitem aos treinados saberem quando aplicar essas habilidades.

\begin{tabular}{|c|c|c|}
\hline Categorias gerais & Dimensões & Características \\
\hline \multirow{3}{*}{ Características Individuais } & $\begin{array}{l}\text { Habilidade } \\
\text { Cognitiva }\end{array}$ & $\begin{array}{l}\text { Pessoas com alta capacidade cognitiva terão maior potencial de aprendizado } \\
\text { nas ações de treinamento. } \\
\text { Condução diferenciada do processo de treinamento para aquelas pessoas com } \\
\text { baixa capacidade cognitiva. }\end{array}$ \\
\hline & Autoeficácia & $\begin{array}{l}\text { Crença de que se pode ter uma performance específica pode levar a um melhor } \\
\text { aprendizado nas ações de treinamento. }\end{array}$ \\
\hline & $\begin{array}{l}\text { Orientação para } \\
\text { metas }\end{array}$ & $\begin{array}{l}\text { Orientação para metas de aprendizagem, caracterizada pela busca de } \\
\text { desenvolvimento de competências por aquisição de novas habilidades. } \\
\text { Orientação para metas de desempenho, na qual o indivíduo procura segurança } \\
\text { de sua própria competência por meio da busca de uma boa avaliação de } \\
\text { desempenho. }\end{array}$ \\
\hline Motivação para treinamento & $\begin{array}{l}\text { Engajamento do } \\
\text { treinado }\end{array}$ & $\begin{array}{l}\text { O esforço, a intensidade, a persistência e a direção que o treinado aplica nas } \\
\text { ações de treinamento orientadas para aprendizagem antes, durante e depois } \\
\text { do processo. } \\
\text { A motivação é importante para o aprendizado e tem implicações diretas no } \\
\text { planejamento e na entrega dos programas de treinamento. }\end{array}$ \\
\hline \multirow[b]{2}{*}{$\begin{array}{l}\text { Condições e Ambiente pré- } \\
\text { treinamento }\end{array}$} & Condições & $\begin{array}{l}\text { Intervenções com informações preparatórias aplicadas antes das ações de } \\
\text { treinamento. }\end{array}$ \\
\hline & $\begin{array}{l}\text { Ambiente e } \\
\quad \text { clima }\end{array}$ & $\begin{array}{l}\text { Relaciona-se com as experiências prévias que os participantes das ações de } \\
\text { treinamento possuem e com a maneira pela qual a organização desenvolve tais } \\
\text { ações. }\end{array}$ \\
\hline
\end{tabular}

Figura 2. Categorias gerais de avaliação das necessidades de treinamento

Fonte: Elaborada pelo autor - adaptado de Salas e Cannon-Bowers (2001). 
Aguinis e Kraiger (2009) afirmaram que as características como envolvimento com o trabalho, comprometimento organizacional, orientação para objetivos de aprendizagem, habilidade cognitiva, autoeficácia, local de controle e percepção do ambiente de trabalho estão relacionadas à motivação prévia com as ações de treinamento e caracterizamse como características individuais e situacionais que devem ser consideradas na fase de avaliação das necessidades das ações de treinamento e melhoram os benefícios dos treinamentos.

Diante do levantamento da produção acadêmica nacional e internacional sobre o tema avaliação de necessidades de treinamento, Abbad e Mourão (2012) propuseram um modelo conceitual de avaliação de necessidade de TD\&E que busca identificar as necessidades de treinamento em uma perspectiva multinível: organizacional, grupal e individual, tendo como base a análise dos desafios e ameaças apresentados pelo ambiente externo, identificando o comportamento dos stakeholders que podem afetar os diversos níveis da organização, causando necessidades de ações educacionais. Na Figura 3 serão apresentadas as diretrizes de avaliação de necessidade de ações educacionais em cada um dos níveis.

A base de avaliação das necessidades de treinamento, apresentada no modelo proposto pelas autoras citadas, considera técnicas e instrumentais que podem pertencer a qualquer um dos domínios de aprendizagem: físico/psicomotor; cognitivo e afetivo, levando-se em conta os processos de desenvolvimento de tais domínios, de forma a considerar um contexto social amplo em suas dimensões econômicas, legais, tecnológicas, políticas, ambientais, educacionais e de saúde pública (Abbad \& Mourão, 2012).

\begin{tabular}{|c|c|c|}
\hline Nível & Foco & Processo metodológico \\
\hline 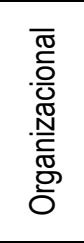 & $\begin{array}{l}\text { Competências transversais ou genéricas } \\
\text { Competências replicáveis }\end{array}$ & $\begin{array}{l}\text { Uso de múltiplas fontes de avaliadores } \\
\text { Questionário eletrônico } \\
\text { Métodos qualitativos e quantitativos de coleta e análise de dados } \\
\text { Aplicação de grupos focais e técnica Delphi } \\
\text { Análise de fluxo de atividade ou mapeamento de processo } \\
\text { Múltiplas fontes documentais e humanas }\end{array}$ \\
\hline 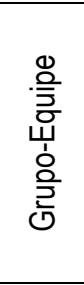 & $\begin{array}{l}\text { Público alvo bem delimitado } \\
\text { Competências emergentes e críticas para } \\
\text { categorias profissionais, cargos, grupos ou } \\
\text { equipes de trabalho } \\
\text { Gaps em competências técnicas específicas }\end{array}$ & $\begin{array}{l}\text { Análise de legislações e normas técnicas da área } \\
\text { Captação de necessidades futuras com especialistas } \\
\text { Exame documental } \\
\text { Indicação de questionários individuais para a clientela } \\
\text { Análise do papel ocupacional } \\
\text { Grupo focal e aplicação de questionário em grupo estruturado ou } \\
\text { semiestruturado, com respostas elaboradas pelo grupo }\end{array}$ \\
\hline 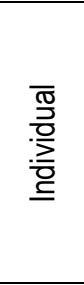 & $\begin{array}{l}\text { Gaps de competências em termos de } \\
\text { conhecimentos, habilidades e atitudes } \\
\text { Objetivos pessoais e profissionais de TD\&E } \\
\text { Competências exigidas a todo profissional } \\
\text { Identificação do perfil do público-alvo } \\
\text { (demográfico, profissional, motivacional e } \\
\text { cognitivo) }\end{array}$ & $\begin{array}{l}\text { Autoavaliação } \\
\text { Aplicação de medidas de percepção de suporte } \\
\text { Análise de contexto organizacional } \\
\text { Análise da magnitude do hiato das competências }\end{array}$ \\
\hline
\end{tabular}

Figura 3. Diretrizes do Modelo Conceitual de Avaliação de Necessidade de Treinamento

Fonte: Elaborada pelo autor, adaptada de Abbad e Mourão (2012).

A partir do referencial teórico apresentado, apresenta-se a seguinte proposição:

$P_{01}$ : A sistematização da avaliação da necessidade de treinamento ambiental pode potencializar 0 desenvolvimento de competências individuais essenciais à sustentabilidade das empresas do setor químico brasileiro.

Após a apresentação da fundamentação teórica sobre a avaliação das necessidades de treinamento, apresentam-se os aspectos metodológicos da pesquisa. 


\section{METODOLOGIA}

Neste trabalho foi utilizada a abordagem de pesquisa qualitativa (Mason, 1996) e a estratégia de pesquisa adotada foi o estudo de casos múltiplos (Yin, 2010). Os critérios de seleção dos casos foram: pertencer ao setor químico brasileiro, sendo membro da Associação Brasileira da Indústria Química (ABIQUIM); ter o Sistema de Gestão do Programa Atuação Responsável da ABIQUIM; ser membro da Comissão do Meio Ambiente da ABIQUIM; e ser signatária do Pacto Global, que é uma iniciativa desenvolvida pela ONU que tem como objetivo a mobilização da comunidade empresarial internacional para a adoção, em suas práticas de negócio, de valores fundamentais e internacionalmente aceitos, nas dimensões de direitos humanos, relações de trabalho, meio ambiente e combate à corrupção, pautadas em dez princípios estabelecidos pelo Pacto Global. Com base nesses critérios, foram estudadas três empresas que serão descritas neste trabalho utilizando nomes fictícios de Alpha (para empresa 1) Ômega (para empresa 2) e Beta (para a empresa 3).

Realizou-se a coleta de dados a partir de pesquisa documental, registro de arquivos e entrevistas em profundidade. Tais técnicas de coleta de dados são fundamentais para a triangulação dos dados coletados, que, segundo Yin (2010), ajuda a abordar problemas potenciais de validade do constructo, pois assim se tem o mesmo fenômeno com várias avaliações por meio de múltiplas fontes de evidência.

Yin (2010), Godoy (2006) e Gil (2009) consideraram que o exame de documentos pode trazer contribuições importantes para o estudo de caso. Complementarmente, a análise de documentos elaborados pela organização passa a ter informações que auxiliam na coleta de dados mediante observação ou entrevista (Gil, 2009). Segundo Yin (2010), os documentos são úteis e seu uso mais importante é para corroborar e aumentar a evidência de outras fontes.

Contextualiza-se que na pesquisa de campo o entrevistador cobriu todas as questões do roteiro sintetizadas. Esse tipo de entrevista, chamada por Gil (2009) de entrevista por pautas, tem como característica o entrevistador fazer poucas perguntas diretas e deixar 0 entrevistado falar livremente, à medida que se refere às pautas assinaladas sem, contudo, deixar que o entrevistado se afaste da pauta.

0 registro dos dados coletados pelas entrevistas foi feito por meio de gravação direta e anotações realizadas pelo pesquisador durante o processo, sendo que esses dados foram, posteriormente, tratados por meio de transcrição das gravações para melhor condição de análise dos dados coletados.

$\mathrm{Na}$ Figura 4 são listados os sujeitos sociais da pesquisa e elencadas as fontes de evidências acessadas em cada empresa.

\begin{tabular}{|c|c|c|c|}
\hline & \multicolumn{3}{|c|}{ EMPRESAS QUE PARTICIPARAM DA PESQUISA } \\
\hline & ALPHA & ÔMEGA & BETA \\
\hline $\begin{array}{l}\text { Natureza do } \\
\text { capital }\end{array}$ & Empresa de capital nacional aberto & $\begin{array}{l}\text { Empresa de capital } \\
\text { estrangeiro aberto }\end{array}$ & Empresa de capital estrangeiro aberto \\
\hline Porte & Grande & Grande & Grande \\
\hline $\begin{array}{c}\text { Ano de } \\
\text { fundação }\end{array}$ & 2002 & 1863 & Final do século XIX \\
\hline $\begin{array}{c}\text { Área e } \\
\text { mercados } \\
\text { de atuação }\end{array}$ & $\begin{array}{l}\text { Atuação nos mercados de } \\
\text { adesivos, agronegócio, borracha, } \\
\text { construção, indústria automotiva, } \\
\text { lubrificantes, tintas e varejo. }\end{array}$ & $\begin{array}{l}\text { Automotivo e aeroespacial, } \\
\text { produtos de consumo e } \\
\text { cuidados de saúde, energia e } \\
\text { meio ambiente, } \\
\text { eletroeletrônico, edificação e } \\
\text { construção e aplicações } \\
\text { industriais. }\end{array}$ & $\begin{array}{l}\text { Transporte, infraestrutura, meio ambiente } \\
\text { e consumidor final. }\end{array}$ \\
\hline 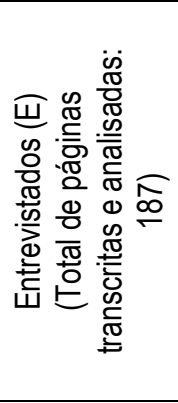 & $\begin{array}{c}\text { - Diretor Desenvolvimento } \\
\text { Sustentável (E1) } \\
\text { - Responsável pela área ambiental } \\
\text { em todos os sites da empresa } \\
\text { Alpha (E2) } \\
\text { - Coordenador de segurança e } \\
\text { meio ambiente das unidades de } \\
\text { negócio - SP (E3) } \\
\text { - Coordenadora de meio ambiente } \\
\text { na UNI2 - RS (E4) }\end{array}$ & $\begin{array}{c}\text { - Gerente de segurança e } \\
\text { meio ambiente corporativo } \\
\text { para a região da América } \\
\text { Latina. (E1) } \\
\text { - Gerente de RH e } \\
\text { responsável na América do } \\
\text { Sul pela área de learning e } \\
\text { Universidade Corporativa } \\
\text { (E2) }\end{array}$ & $\begin{array}{c}\text { - Gerente de SSMA (E1) } \\
\text { - Coordenadora de Sistema de Gestão } \\
\text { (E2) } \\
\text { - Engenheira de Meio Ambiente e } \\
\text { Segurança (E3) } \\
\text { - Analista de RH - encarregada de } \\
\text { treinamento (E4) }\end{array}$ \\
\hline
\end{tabular}




\begin{tabular}{|c|c|c|c|}
\hline 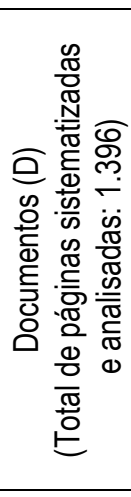 & $\begin{array}{c}\text { - Apresentação sobre Gestão } \\
\text { Ambiental } 2015 \text { (D1) } \\
\text { - Modelo de plano de aula de } \\
\text { educação ambiental (D2) } \\
\text { - Apresentação utilizada no } \\
\text { Workshop de Líderes para o } \\
\text { Desenvolvimento Sustentável (D3) } \\
\text { - Relatório Anual } 2015 \text { (D4) } \\
\text { - Dados disponíveis no site } \\
\text { institucional da empresa (D5) }\end{array}$ & $\begin{array}{c}\text { - Global Report } 2015 \text { (D1) } \\
\text { - Relatório Anual } \\
\text { Complementar } 2015 \text { (D2) } \\
\text { - Global Report } 2013 \text { (D3) } \\
\text { - Mapas de competências } \\
\text { (D4) } \\
\text { - Dados disponíveis no site } \\
\text { institucional da empresa (D5) }\end{array}$ & $\begin{array}{l}\text { - Relatório de Sustentabilidade 2014/5 } \\
\text { (D1) } \\
\text { - Atualização do Relatório de } \\
\text { Sustentabilidade } 2015 \text { (D2) } \\
\text { - Política de Saúde, Segurança e Meio } \\
\text { Ambiente (D3) } \\
\text { - Programa da Semana do Meio } \\
\text { Ambiente } 2016 \text { (D4) } \\
\text { - Lista de treinamentos de Meio Ambiente } \\
\text { (D5) } \\
\text { - Dados disponíveis no site institucional } \\
\text { da empresa (D6) }\end{array}$ \\
\hline 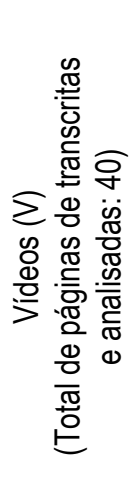 & $\begin{array}{l}\text { - Vídeo da entrevista do diretor de } \\
\text { desenvolvimento sustentável da } \\
\text { empresa Alpha no IV Sarau de } \\
\text { Negócios da Editora Atlas. (V1) } \\
\text { - Vídeo do depoimento do vice- } \\
\text { presidente de relações } \\
\text { institucionais e desenvolvimento } \\
\text { sustentável da empresa Alpha. (V2) } \\
\text { - Vídeo do "Seminário de Educação } \\
\text { Ambiental empresa Alpha". (V3) }\end{array}$ & $\begin{array}{l}\text { - Vídeo de apresentação do } \\
\text { programa ÔMEGA Way (V1) } \\
\text {-Vídeo de entrevista de CEO } \\
\text { da empresa Ômega e de um } \\
\text { executivo da empresa sobre } \\
\text { as ferramentas do programa } \\
\text { de gestão da } \\
\text { sustentabilidade. (V2) } \\
\text { - Vídeo do CEO da empresa } \\
\text { Ômega tratando sobre a } \\
\text { questão de emissão e crédito } \\
\text { de carbono (V3) }\end{array}$ & $\begin{array}{l}\text { - Vídeo com depoimento de três altos } \\
\text { executivos da empresa sobre } \\
\text { desenvolvimento sustentável (V1) } \\
\text { - Vídeo com depoimento do presidente } \\
\text { da empresa sobre meio ambiente (V2) }\end{array}$ \\
\hline
\end{tabular}

Figura 4. Caracterização das fontes de evidências das empresas pesquisadas.

Fonte: Elaborada pelos autores.

A análise dos dados foi feita a partir da categoria avaliação da necessidade de treinamento com base em suas dimensões e propriedades, conforme apresentado no Figura 5.

\begin{tabular}{|c|c|c|}
\hline DIMENSÃO & PROPRIEDADES & AUTORES \\
\hline ABRANGÊNCIA & Nível organizacional & Aguinis e Kraiger (2009) \\
DOS & Nível grupal & Abbad e Mourão (2012) \\
GAPS DE & Nindividual & Narashimhan e Ramanarayanan (2014) \\
COMPETÊNCIA & Reativa, não envolvendo os objetivos & Arshad et al. (2015) \\
ABORDAGEM & estratégicos da organização. \\
& Proativa, considerando os intentos estratégicos \\
da organização. & \\
\hline
\end{tabular}

Figura 5. Síntese das dimensões e propriedades do processo de avaliação de necessidades de treinamento Fonte: Elaborada pelo autor baseada nos autores citados.

Vale destacar que a análise dos resultados foi norteada pelos constructos "treinamento ambiental", "competência organizacional" e "competência individual", a partir de uma reorganização teórica em categorias e dimensões levantadas pela literatura, conforme consubstanciado pelas evidências do campo descritas e analisadas no próximo item.

\section{APRESENTAÇÃO E DISCUSSÃO DOS RESULTADOS}

0 construto treinamento ambiental foi analisado com base na categoria que envolve sua fase: 'avaliação da necessidade de treinamento' (ANT) que será apresentada a seguir com base em suas dimensões, sendo elas: abrangência dos gaps de competência e abordagens para a categoria ANT. 
Ao se analisar a categoria 'análise das necessidades de treinamento' e, especificamente, a dimensão da abordagem dos gaps de competência, ficaram evidenciadas as propriedades organizacional, grupal e individual. A análise dessa categoria é apresentada na Figura 6 a partir dos trechos das entrevistas.

\begin{tabular}{|c|c|c|c|c|}
\hline & Nível & Descrição & Empresa & Fragmentos entrevistas \\
\hline & & 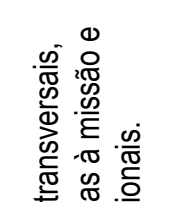 & $\underset{\frac{1}{1}}{\frac{3}{2}}$ & $\begin{array}{l}\text { "Existe uma estratégia que é a da empresa, é corporativa. E aí, nós focamos } \\
\text { em } 10 \text { temas e dentro desses } 10 \text { temas se foca o desenvolvimento da } \\
\text { empresa." (E1). "Nós temos algumas diretrizes dizendo por exemplo alguns } \\
\text { temas críticos que as pessoas devem treinamento, devem se desenvolver." } \\
\text { (E2). }\end{array}$ \\
\hline & 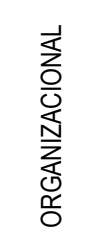 & 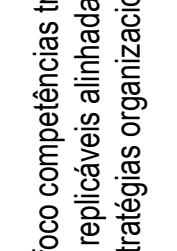 & 志 & $\begin{array}{l}\text { "Eu diria que hoje está vindo muita coisa da corporação. Evidente que } \\
\text { localmente, como região ou como país, a gente pode ter necessidades mais } \\
\text { específicas, mas, atualmente, eu diria para você que não. (E1) } \\
\text { "Foi criada uma dimensão mundial para a sustentabilidade, diretamente } \\
\text { ligada ao CEO do grupo, né, então essa direção mundial ela é responsável } \\
\text { por construir todo o programa que tem de ser desdobrado internamente" (E1) }\end{array}$ \\
\hline & & 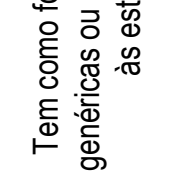 & 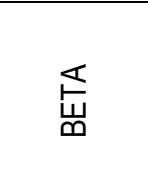 & $\begin{array}{l}\text { "A corporação pede para a gente fazer uma vez por ano para mesmo } \\
\text { relembrar [...] todos os aspectos e impactos ambientais que a empresa possui. } \\
\text { " (E4) }\end{array}$ \\
\hline & & 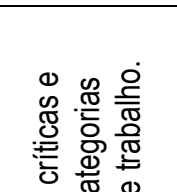 & 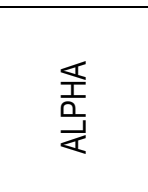 & $\begin{array}{l}\text { "A função dele é x, ele é operador, então ele precisa conhecer esse, esse, } \\
\text { esse, tema, esse tema ele vai ser treinado" (E2). "Pegamos um desvio em } \\
\text { relação a resíduo, vamos trabalhar esse tópico. " (E4). }\end{array}$ \\
\hline 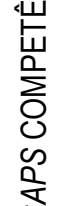 & 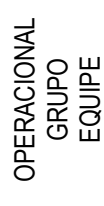 & 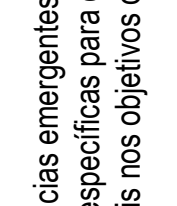 & 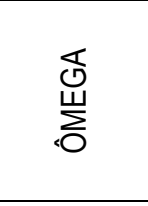 & $\begin{array}{l}\text { "[...] uma determinada área pode chegar a uma conclusão que ela está } \\
\text { precisando reforçar uma sensibilização ou reforçar uma orientação às equipes } \\
\text { nesse item" (E2) } \\
\text { "Na avaliação de desempenho o líder e o liderado sentam e vão discutir as } \\
\text { necessidades da sua equipe." (E2) }\end{array}$ \\
\hline & & 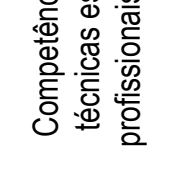 & 䓌 & $\begin{array}{l}\text { "Vamos supor que eu observo que o pessoal da manutenção não está } \\
\text { seguindo adequadamente algum procedimento de meio ambiente, então a } \\
\text { gente vai lá e mesmo que já tenha passado pelo treinamento naquele ano, a } \\
\text { gente reforça os conceitos" (E3) }\end{array}$ \\
\hline & & 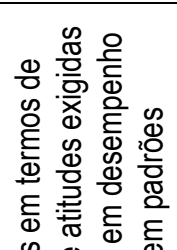 & 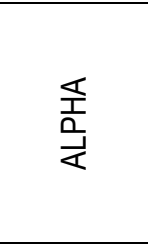 & $\begin{array}{l}\text { "A gente avalia através das entregas, das metas. " (E2). "Também associado } \\
\text { ao plano de desenvolvimento individual da pessoa e ao plano de carreira da } \\
\text { pessoa." (E2). "A gente faz auditoria no dia a dia, assim, como as pessoas } \\
\text { estão agindo" (E4). "A pessoa, em uma relação líder-liderado, identifica o que } \\
\text { aquela pessoa que tem aquele desafio precisaria ampliar em termos de } \\
\text { conhecimento para que aquele desafio seja alcançado" (E1) }\end{array}$ \\
\hline & 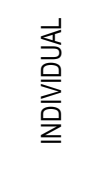 & 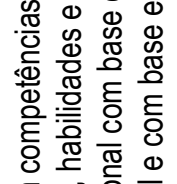 & 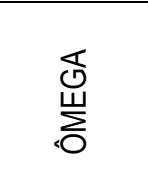 & $\begin{array}{l}\text { "Acontece no âmbito individual, o líder avalia seu liderado [...] o líder entra, vê } \\
\text { os } 10 \text { liderados dele; os respectivos RHs também têm acesso, ou seja, essas } \\
\text { avaliações servem de inputs para o próximo plano de desenvolvimento" (E2) }\end{array}$ \\
\hline & & 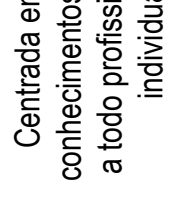 & 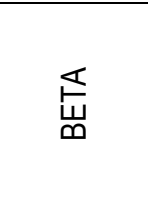 & $\begin{array}{l}\text { "Dependendo da atividade que ele vai exercer ele tem os treinamentos } \\
\text { relacionados" (E2) } \\
\text { "A liderança tem pelo menos uma vez por mês de ir para a área, acompanhar } \\
\text { algum trabalho que está em andamento e verificar várias questões, e tem } \\
\text { questões de segurança e questões de meio ambiente." (E3) }\end{array}$ \\
\hline
\end{tabular}

Figura 6. Dados sobre Abordagens da ANT

Fonte: Elaborada pelo autor.

Na empresa Alpha, ao se analisar a categoria 'avaliação da necessidade de treinamento' em sua dimensão da abrangência dos gaps de competências, constatou-se, no que diz respeito à propriedade do nível organizacional, que há foco em competências transversais alinhadas aos objetivos estratégicos que se busca desenvolver na organização. Tal constatação foi encontrada na fala dos entrevistados (E1) e (E2), bem como na análise documental com evidência de treinamentos desenvolvidos por meio de um Programa de Desenvolvimento de Competências em Sustentabilidade, 
adotado pela empresa desde 2012. A análise do conteúdo do (D1) associa a necessidade de treinamento aos objetivos estratégicos ao colocar em destaque como objetivo do workshop: "apresentar fundamentos teóricos e fomentar discussões sobre o Desenvolvimento Sustentável (DS) que possam fortalecer a estratégia de DS e contribuir para 0 alcance da estratégia de longo prazo da Alpha".

A estratégia corporativa da empresa fornece direcionamento dos temas críticos que devem ser desenvolvidos pelos programas de treinamento, o que corrobora Narashimhan e Ramanarayanan (2014), ao afirmarem que a missão e as estratégias da organização caracterizam a avaliação da necessidade de treinamento na abordagem organizacional, bem como Aguinis e Kraiger (2009), para quem as competências centrais da organização integram tanto a estratégia quanto as rotinas e as práticas da organização.

No que diz respeito às propriedades de nível operacional, evidenciou-se nas falas dos entrevistados que os gaps de competências encontrados no nível de grupo/equipes fornecem elementos para avaliação da necessidade de treinamento. $O$ entrevistado (E2) associa as funções, enquanto o entrevistado (E3) associa a contribuição à avaliação da necessidade de treinamento às áreas que irão definir necessidades específicas. A entrevistada coordenadora do meio ambiente na UNI2 (E4) mostra evidência de que gaps em temas específicos demonstram necessidades de treinamentos. Essas evidências estão alinhadas aos autores Abbad e Mourão (2012), bem como Narashimhan e Ramanarayanan (2014), para quem o foco da ANT também deve ser direcionado a gaps de competências emergentes e críticas para cargos, grupos ou áreas.

A dimensão individual, associada ao desempenho individual com base em padrões estabelecidos, mostrou-se presente na empresa Alpha na fala dos entrevistados. O entrevistado (E2) associa as entregas ao plano de desenvolvimento individual e ao plano de carreira da pessoa. A entrevistada (E4) identifica a importância da avaliação comportamental que está alinhada às competências em termos de atitudes em relação ao que se é exigido da pessoa. 0 entrevistado (E1) descreve a importância da relação líder-liderado no processo de identificação dos gaps de competências individuais a serem desenvolvidos. Os dados coletados mostram-se alinhados à base teórica desenvolvida por Abbad $\mathrm{e}$ Mourão (2012) e Narashimhan e Ramanarayanan (2014), quando o foco da ANT se direciona ao gap de competências em termos de conhecimento, habilidades e atitudes ou dos objetivos pessoais e profissionais do indivíduo.

$\mathrm{Na}$ empresa Ômega, a avaliação das necessidades de treinamento é evidenciada nos três níveis organizacionais, e com foco na estratégia da organização. No que tange ao nível organizacional, as evidências mostram que as diretrizes que definem os objetivos rumo à sustentabilidade, conforme afirmado pelos entrevistados, seguem um direcionamento global, no sentido de serem estabelecidas diretrizes mundiais que devem ser seguidas pela organização. Não obstante as regiões contarem com condições de desenvolver o seu sistema de treinamento, o direcionamento é corporativo, alinhado à base teórica de Narashimhan e Ramanarayanan (2014), que expõem a dimensão organizacional alinhada às estratégias da organização como fonte de caracterização das necessidades de treinamento.

No que diz respeito ao nível operacional, os gaps de competência são evidenciados em nível de área e de equipes. $O$ nível individual também se mostra presente no levantamento dos gaps de competência, por meio das avaliações de desempenho que são realizadas e alimentam a área de gestão de pessoas de informações, no que diz respeito às necessidades de treinamento. Tais evidências corroboram as propriedades teóricas desenvolvidas por Narashimhan e Ramanarayanan (2014).

$\mathrm{Na}$ empresa Beta, a identificação de lacunas de competências voltadas para o trabalho ou para o atendimento dos objetivos organizacionais é um componente essencial do processo do treinamento. Segundo Narashimahan e Ramanarayanan (2014), que corroboram Ramus (2002) e Jabbour et al. (2010), a avaliação da necessidade de treinamento pode abranger três dimensões: aquela oriunda da missão e dos objetivos estratégicos; a que contribui para que se alcançem os objetivos de cada trabalho; e a proveniente do gap do desempenho do indivíduo frente aos padrões estabelecidos.

Todas essas dimensões foram identificadas nas evidências encontradas na empresa Beta pelas entrevistas e a dimensão organizacional também foi evidenciada pelos documentos (D1), quando afirma que o desenvolvimento das competências é o fator-chave para o plano de carreira do funcionário, bem como os documentos (D2), (D3) e (D4), de modo que os dados corroboram a abordagem teórica de que a avaliação da necessidade de treinamento é uma fase do processo que incorpora as três esferas de abrangência.

Após apresentação e discussão das evidências encontradas nas empresas estudadas, sintetizam-se as propriedades descritoras na Avaliação das Necessidades de Treinamento da categoria ANT no que diz respeito à abrangência dos níveis de gaps de competência, conforme apresentado na Figura 7. 


\begin{tabular}{|c|c|c|c|}
\hline & \multicolumn{3}{|c|}{ Abrangência dos níveis de gaps de competências } \\
\hline & Organizacional & Operacional & Individual \\
\hline$\frac{\text { 을 }}{\frac{\text { 은 }}{2}}$ & $\begin{array}{l}\text { Envolve as competências } \\
\text { alinhadas aos intentos estratégicos } \\
\text { corporativos, direcionados pelos } \\
\text { macro-objetivos organizacionais } \\
\text { para 2020. }\end{array}$ & $\begin{array}{l}\text { Inclui as necessidades de } \\
\text { desenvolvimento de competências } \\
\text { das áreas ou das unidades que } \\
\text { possuem especificidades no que } \\
\text { diz respeito às suas operações em } \\
\text { relação aos impactos ambientais. }\end{array}$ & $\begin{array}{l}\text { Aborda a avaliação das entregas realizadas } \\
\text { pelas pessoas da organização, por meio da } \\
\text { avaliação de desempenho, bem como o } \\
\text { plano de desenvolvimento individual que } \\
\text { vai caracterizar as competências } \\
\text { necessárias ao indivíduo. }\end{array}$ \\
\hline 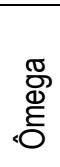 & $\begin{array}{l}\text { Envolve as necessidades de } \\
\text { treinamento alinhadas à busca do } \\
\text { atendimento aos objetivos } \\
\text { estratégicos. }\end{array}$ & $\begin{array}{l}\text { Inclui os gaps de competência de } \\
\text { áreas ou equipes para alcançar a } \\
\text { realização dos objetivos de } \\
\text { trabalho. }\end{array}$ & $\begin{array}{l}\text { Aborda a necessidade de desenvolvimento } \\
\text { da pessoa para realização de suas } \\
\text { entregas alinhadas aos padrões } \\
\text { estabelecidos. }\end{array}$ \\
\hline$\frac{T}{\Phi}$ & $\begin{array}{l}\text { Envolve a busca por melhorar } 0 \\
\text { desempenho organizacional. }\end{array}$ & $\begin{array}{l}\text { Inclui os conhecimentos e as } \\
\text { competências necessárias para as } \\
\text { áreas e equipes de trabalho }\end{array}$ & $\begin{array}{l}\text { Aborda os gaps de competências } \\
\text { identificados nos indivíduos na execução } \\
\text { de seu trabalho. }\end{array}$ \\
\hline
\end{tabular}

Figura 7. Abrangências dos níveis dos gaps de competência

Fonte: Elaborada pelo autor.

No que tange aos níveis de análise dos gaps de competências, nas três empresas estudadas houve alinhamento com a teoria em relação a todos os níveis de análise: organizacional, operacional e individual.

O nível organizacional é identificado em cada uma das empresas tanto pelas demandas corporativas, tais como aumento da receita proveniente de produtos que atendam às necessidades dos clientes e diminuam os impactos ambientais, redução da emissão dos gases de efeito estufa, uso de matérias-primas renováveis, dentre outras que são definidas a partir dos objetivos estratégicos globais, como eficiência hídrica, eficiência energética, redução de geração de resíduos, reconhecimento em termos de segurança, quanto por demandas de unidades de negócios que podem ter características específicas em termos de necessidades em relação à sua linha de produção, como maior demanda de recursos hídricos, maior geração de resíduos sólidos, maior consumo energético, gerando, assim, necessidade de competências em nível organizacional, alinhadas às particularidades de cada negócio.

A 'avaliação das necessidades de treinamento' no nível operacional, ou também chamado por alguns autores de grupos e equipes, foi também evidenciada nas três empresas estudadas, sendo caracterizada por grupo de funções, por área funcional ou, até mesmo, por atividades específicas relacionadas ao meio ambiente, destacando-se: a área de gestão ambiental, de manutenção, de pesquisa e desenvolvimento.

A mesma situação de alinhamento foi encontrada nas empresas no que diz respeito à avaliação da necessidade de treinamento no nível individual. Em todas as empresas estudadas as fontes pelas quais são evidenciadas as necessidades de treinamento na dimensão individual são avaliação de desempenho e plano de desenvolvimento pessoal ou plano de carreira. A empresa Alpha destacou também, além dessas duas fontes, as auditorias internas que são realizadas pelo processo da ISO 14001.

Os dados encontrados nas três empresas estudadas, além de corroborarem os autores que indicam a necessidade da avaliação de necessidade de treinamento nos três níveis de abrangência como Aguinis e Kraiger (2009), Abbad e Mourão (2012) e Narashimhan e Ramanarayanam (2014), também corroboram a base teórica estabelecida por Jabbour et al. (2010), que mapearam, dentre as melhores práticas de treinamento ambiental, a avaliação de necessidade de treinamento que deve considerar o impacto das responsabilidades dos empregados em todos os níveis organizacionais, de modo a estimular a motivação dos empregados no que se refere às questões ambientais; quanto Ramus (2002), Unnikrishnam e Hedge (2007), Daily et al. (2012), Mandip (2012) e Jabbour (2013), que estacaram como principais fatores do treinamento ambiental sua abrangência nos níveis estratégico, tático e operacional de modo que esteja presente em toda a organização. Os dados estão alinhados também à literatura, na qual as práticas de gestão de pessoas têm um papel importante no desenvolvimento da sustentabilidade organizacional, tendo como destaque 0 sistema de avaliação de desempenho e o plano de carreira (Daily \& Huang, 2001; Jabbour et al., 2010a). 


\begin{tabular}{|c|c|c|c|c|}
\hline \multirow{7}{*}{ 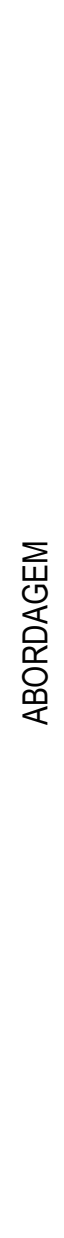 } & & Descrição & Empresa & Fragmentos entrevistas \\
\hline & \multirow{3}{*}{ 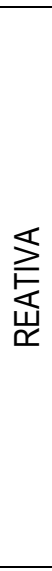 } & \multirow{3}{*}{ 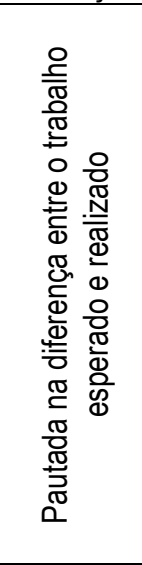 } & $\begin{array}{l}\text { 罙 } \\
\frac{1}{2}\end{array}$ & $\begin{array}{l}\text { "Eu aponto esse problema na auditoria, o pessoal faz essa análise crítica e } \\
\text { normalmente, dentro das ações corretivas envolve o treinamento das pessoas. " (E2). } \\
\text { "Esses desvios são verificados e aí a gente trabalha para fortalecer. " (E4) }\end{array}$ \\
\hline & & & 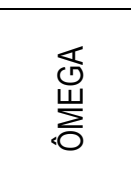 & $\begin{array}{l}\text { "Nosso levantamento de necessidade de treinamento que é feito dentro do nosso } \\
\text { processo de avaliação de desempenho. (E2) }\end{array}$ \\
\hline & & & 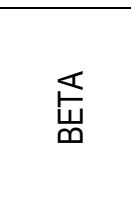 & $\begin{array}{l}\text { "Quando a gente fala da parte ambiental, o primeiro item que vem como estrutura é } \\
\text { ISO14000" (E2) } \\
\text { "Coloca ali no papel quais foram os gaps do ano anterior, o que precisa para } \\
\text { melhorar, colocando para aqueles objetivos também, o que a gente precisa agregar." } \\
\text { (E4) }\end{array}$ \\
\hline & \multirow{3}{*}{ 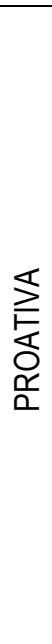 } & \multirow{3}{*}{ 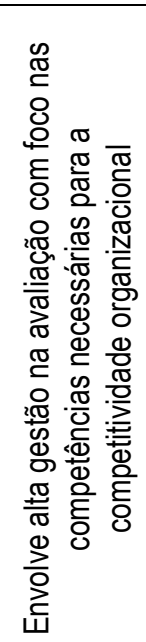 } & $\begin{array}{l}\text { 䍃 } \\
\frac{1}{<}\end{array}$ & $\begin{array}{l}\text { "Nós temos algumas diretrizes dizendo, por exemplo, alguns temas críticos que as } \\
\text { pessoas devem se desenvolver. " (E2) "Para você ter uma ideia, nós tivemos que } \\
\text { aprender a fazer a gestão de risco ou identificação de riscos decorrente das } \\
\text { mudanças climáticas para 2040. Isso não é uma competência que nós dominávamos, } \\
\text { tivemos que capacitar pessoas para isso e hoje conseguimos identificar quais são } \\
\text { esses riscos. Isso é uma competência assim mais estratégica." (E1) }\end{array}$ \\
\hline & & & 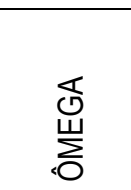 & $\begin{array}{l}\text { "O grupo Ômega vem gerando bastante formação, treinamento de forma corporativa, } \\
\text { e focado realmente nas grandes necessidades do grupo. Por exemplo, esse programa } \\
\text { Sustentabilidade, saiu um pouco disso. O pessoal lançou todo esse programa, foi a } \\
\text { alta direção que criou essa ferramenta corporativa, mas também criou formação" (E1) }\end{array}$ \\
\hline & & & 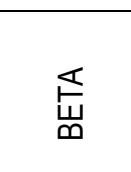 & $\begin{array}{l}\text { "A gente está participando do Atuação Responsável, também pela ABIQUIM, então a } \\
\text { gente precisa de um treinamento" (E4) } \\
\text { "A empresa tem um programa de gerenciamento de perigos e risco de processo, } \\
\text { segurança de processo" (E1) }\end{array}$ \\
\hline
\end{tabular}

Figura 8. Abordagens reativa e proativa

Fonte: Elaborada pelo autor

Ao se analisar a categoria 'análise das necessidades de treinamento' e, especificamente, a dimensão das abordagens utilizadas, constataram-se as propriedades reativas e proativas presentes no processo de análise das necessidades de treinamento, conforme Figura 8, a partir de trechos de entrevistas selecionadas.

$\mathrm{Na}$ empresa Alpha, com a análise da dimensão 'abordagens adotadas' da categoria 'avaliação da necessidade de treinamento', evidenciou-se que tanto a abordagem em sua propriedade reativa quanto proativa são utilizadas.

A abordagem reativa é manifestada na fala dos entrevistados, quando a associam principalmente à diferença entre o desempenho realizado e o esperado. Tal abordagem é associada principalmente pelos processos de auditorias em que desvios são encontrados por meio de não conformidades identificadas no processo de auditoria interna realizada pela empresa. Essas evidências foram encontradas nas falas dos entrevistados (E2) e (E4).

Evidenciou-se também a abordagem reativa na fala do entrevistado (E2) associada à exigência legal e, não necessariamente, com foco nas competências necessárias para a competitividade da empresa. Essa abordagem tem um papel caracterizado mais pelo controle do que propriamente estratégico para a organização. Tais evidências podem ser caracterizadas como reativa, em função de ser a necessidade de treinamento oriunda da diferença entre o desempenho esperado e o realizado, ou da busca por cumprimento à legislação e não dos objetivos estratégicos, conforme conceitualizado por Ashad et al. (2015).

A abordagem proativa, caracterizada pelo envolvimento da alta gestão e pela necessidade de competências oriunda dos intentos estratégicos fundamentais para a competitividade da organização (Arshad et al., 2015), foi evidenciada e corroborada na fala dos sujeitos entrevistados nessa empresa. Constatou-se que a organização estabelece diretrizes alinhadas à sua estratégia organizacional, as quais direcionam a avaliação da necessidade de treinamento.

$O$ entrevistado (E4) confirma tal evidência, ao dizer que há treinamentos e que todos da empresa precisam fazer. O entrevistado (E2) destaca temas críticos, tais como gestão de resíduos, gestão e efluentes, controle de emissões, que 
compõem a necessidade de treinamento, que são definidos pelas diretrizes organizacionais. $O$ entrevistado (E1) ilustra a necessidade de desenvolvimento de competências estratégicas que motivaram a importância de aprendizagem em temas específicos relacionados ao meio ambiente, como tratamento de resíduo pós consumo de plástico, gestão de risco associado a produtos químicos. Os documentos (D1) e (D2) também evidenciam tal abordagem, tanto pelo objetivo e desenvolvimento do workshop, quanto pelo alinhamento do plano de aula aos 10 macro-objetivos estratégicos apresentados no documento (D4), como: reconhecimento por sua contribuição para a reciclagem de plásticos; ter a liderança reconhecida pela fabricação de produtos químicos a partir de matérias-primas renováveis; continuar sendo referência em uso de recursos hídricos na indústria química mundial; estar entre as melhores empresas do mundo em intensidade de emissões de gases do efeito estufa. Essa abordagem proativa caracteriza um papel estratégico da ANT, uma vez que busca estar alinhada aos objetivos estratégicos da organização.

Com base nos dados empíricos, evidenciou-se que a categoria de ANT na empresa Alpha apresenta como objetivo o levantamento de necessidade de treinamento pautado nos gaps de competências que abrangem os níveis organizacional, grupal e individual tanto em uma abordagem reativa quanto em proativa.

$\mathrm{Na}$ empresa Ômega, no que concerne às abordagens, evidenciou-se a presença da abordagem reativa alinhada à dimensão do indivíduo, pautada na avaliação de desempenho que fornece elementos de necessidade de treinamento, pela diferença entre o que a pessoa está entregando e o que é esperado dela. Também a perspectiva proativa foi identificada como utilizada na ANT com foco nos intentos estratégicos e na dimensão corporativa que estabelece as diretrizes de treinamento direcionadas aos objetivos estratégicos definidos pela alta administração. Dessa forma, as abordagens utilizadas corroboram o exposto por Arshad et al. (2015).

A avaliação da necessidade de treinamento, analisada a partir das abordagens estabelecidas por Arshad et al. (2012), também foi corroborada pelas evidências encontradas nos dados coletados da empresa Beta. A abordagem proativa, que representa as necessidades de treinamento pautadas nas diretrizes estratégias da organização, foi encontrada nos dados de entrevistas dos entrevistados (E1) e (E4), bem como nos documentos (D1), (D2) e (D3).

No que tange à abordagem reativa pautada nos gaps identificados com base do desempenho dos indivíduos, frente aos padrões estabelecidos, foi também identificada pelo entrevistados (E1) e (E4) e pelo documento (D1).

A partir das evidências analisadas, pode-se sistematizar as abordagens presentes em cada uma das empresas estudadas, conforme apresentado na Figura 9.

\begin{tabular}{|c|c|c|}
\hline & Reativa & Proativa \\
\hline$\frac{\text { 而 }}{\frac{1}{1}}$ & $\begin{array}{c}\text { Envolve tanto uma avaliação entre o desempenho } \\
\text { esperado e realizado, quanto a busca da } \\
\text { identificação do que é necessário para o } \\
\text { cumprimento da legislação. }\end{array}$ & $\begin{array}{l}\text { Inclui o envolvimento da alta administração na definição das } \\
\text { necessidades a serem trabalhadas para se alcançar os objetivos } \\
\text { estratégicos em termos de resultados organizacionais. }\end{array}$ \\
\hline 莺 & $\begin{array}{c}\text { Envolve a identificação de necessidade de } \\
\text { capacitação visando à correção dos resultados } \\
\text { entregues pelos indivíduos diante dos padrões } \\
\text { estabelecidos. }\end{array}$ & $\begin{array}{l}\text { Inclui a necessidade de desenvolvimento de treinamentos que } \\
\text { estejam alinhados aos objetivos estratégicos da organização. }\end{array}$ \\
\hline $\begin{array}{l}\frac{\pi}{4} \\
\infty\end{array}$ & $\begin{array}{c}\text { Envolve a identificação da necessidade de } \\
\text { treinamento pautado na diferença entre os padrões } \\
\text { estabelecidos e as entregas dos indivíduos na } \\
\text { organização. }\end{array}$ & $\begin{array}{l}\text { Inclui o estabelecimento de necessidades de treinamentos a } \\
\text { partir dos objetivos estratégicos da organização. }\end{array}$ \\
\hline
\end{tabular}

Figura 9. Sistematização das abordagens reativa e proativa nas empresas estudadas

Fonte: Elaborada pelo autor.

Com base nos dados coletados, analisa-se que a ANT é uma fase do processo do treinamento que deve ser feita levando-se em consideração as dimensões organizacional, grupal e individual, bem como se utilizando tanto uma abordagem reativa que leva em consideração os gaps identificados entre o desempenho e os padrões estabelecidos, quanto a abordagem proativa que estabelece as diretrizes estratégicas em uma perspectiva de desenvolvimento de competências necessárias ao alcance da visão organizacional. 
A segunda dimensão estudada, em relação à 'avaliação das necessidades de treinamento', foi a abordagem utilizada pelas empresas. Os dados mostram que as três empresas estudadas utilizam as duas abordagens: a reativa e a proativa. A primeira, em que as necessidades de treinamento são identificadas a partir do diagnóstico de gaps entre 0 desempenho esperado e o realizado pelo funcionário ou pela equipe, feito, principalmente, pela avaliação de desempenho e pelos resultados de auditorias associadas à estrutura da ISO 140001. A abordagem proativa está presente nas empresas por meio das necessidades de treinamento levantadas a partir das diretrizes estratégicas organizacionais, associadas à dimensão ambiental que envolve os programas direcionados ao desenvolvimento de competências para a sustentabilidade, os quais são elaborados em uma dimensão corporativa. Os dados empíricos evidenciados encontrados nas três empresas pesquisadas mostram que elas analisam a necessidade de treinamento tanto a partir da identificação de gaps entre o resultado esperado e 0 realizado pelos funcionários, quanto com base nos objetivos estratégicos organizacionais, corroborando a base teórica estabelecida por Arshad et al. (2015), que afirmam a existência de duas abordagens que podem ser utilizadas na avaliação das necessidades de treinamento: reativa e proativa.

A partir da apresentação dos dados de cada empresa separadamente, passa-se à apresentação e à discussão dos dados agrupados conforme síntese apresentada na Figura 10.

\begin{tabular}{|c|c|c|c|c|}
\hline \multirow{2}{*}{ Categorias de análise } & \multirow{2}{*}{ Dimensões } & \multicolumn{3}{|c|}{ Propriedades descritoras } \\
\cline { 3 - 5 } & ALPHA & ÓMEGA & BETA \\
\hline $\begin{array}{c}\text { AVALIAÇÃO DAS } \\
\text { NECESSIDADES DE } \\
\text { TREINAMENTO }\end{array}$ & \begin{tabular}{c} 
Absangencia dos \\
\cline { 2 - 5 }
\end{tabular} & $\begin{array}{c}\text { Organizacional } \\
\text { Operacional } \\
\text { Individual }\end{array}$ & $\begin{array}{c}\text { Organizacional } \\
\text { Operacional } \\
\text { Individual }\end{array}$ & $\begin{array}{c}\text { Organizacional } \\
\text { Operacional } \\
\text { Individual }\end{array}$ \\
\cline { 2 - 5 } & Abordagens & $\begin{array}{c}\text { Reativa } \\
\text { Proativa }\end{array}$ & $\begin{array}{c}\text { Reativa } \\
\text { Proativa }\end{array}$ & $\begin{array}{c}\text { Reativa } \\
\text { Proativa }\end{array}$ \\
\hline
\end{tabular}

Figura 10. Síntese da discussão agregada dos casos.

Fonte: Elaborada pelos autores.

Com base nas dimensões analisadas da categoria 'avaliação das necessidades de treinamento', pode-se afirmar que, pelos dados coletados nas empresas estudadas, é importante que essa avaliação seja feita tendo como foco os três niveis organizacionais: individual, grupal e organizacional e que se utilize não somente uma abordagem de corrigir desvios encontrados entre os resultados esperados e realizados, abordagem reativa, mas também que se busque diagnosticar as necessidades que tenham como foco uma dimensão de futuro, de desenvolver as competências necessárias para 0 estabelecimento da sustentabilidade ambiental como uma vantagem competitiva para a empresa com foco no longo prazo, utilizando a abordagem proativa.

A partir dos resultados alcançados, pode-se confirmar a proposição $P 01$ de que a sistematização da avaliação da necessidade de treinamento ambiental pode potencializar o desenvolvimento de competências individuais essenciais à sustentabilidade das empresas do setor químico brasileiro, considerando-se que a avaliação da necessidade de treinamento ambiental, por meio de sua abrangência individual, grupal e organizacional e da utilização da abordagem proativa, busca identificar necessidade de treinamentos ambientais que possam contribuir para o desenvolvimento de competências alinhadas às competências para sustentabilidade das empresas do setor químico brasileiro a partir dos gaps de competências identificados.

Com isso, finaliza-se a discussão teórico-empírica no que diz respeito à categoria avaliação da necessidade de treinamento ambiental.

\section{CONSIDERAÇÕES FINAIS}

A avaliação da necessidade de treinamento, pautada na análise dos gaps de competências individuais, grupais e organizacionais, mostra a importância que o treinamento ambiental tem em relação ao mapeamento das competências e do alinhamento dessas competências às estratégias organizacionais no que tange à dimensão ambiental da sustentabilidade.

As abordagens utilizadas para a realização da 'avaliação da necessidade de treinamento', reativa e proativa, mostraram-se importantes no direcionamento das ações. A abordagem reativa aponta a necessidade de correção de curso, no que tange à dimensão ambiental da sustentabilidade, caso desvios em relação aos objetivos a serem 
alcançados sejam evidenciados. A abordagem proativa busca uma perspectiva de evolução, uma vez que seu foco recai em desenvolver competências necessárias aos objetivos de longo prazo que as empresas desenvolvem no que diz respeito às suas estratégias de sustentabilidade ambiental. Desse modo, a utilização, pelas empresas pesquisadas, da abordagem proativa caracteriza que se busca avaliar necessidade de treinamento visando ao desenvolvimento de competências, a fim de responder às exigências essenciais para a competitividade organizacional no que diz respeito à sustentabilidade.

A análise da avaliação da necessidade de treinamento, considerando a abrangência dos gaps de competências nos níveis individual, grupal e organizacional e as abordagens reativa e proativa, aponta também para a necessidade tanto de integração entre os subsistemas da área de gestão de pessoas, como de avaliação de desempenho e de plano de carreira ou desenvolvimento pessoal; quanto de alinhamento destes subsistemas aos intentos estratégicos, no que diz respeito à sustentabilidade.

As contribuições que poderão advir deste estudo para o contexto organizacional estão relacionadas à descrição da fase de avaliação da necessidade de treinamento ambiental desenvolvida pelas empresas estudadas, podendo servir de benchmark para outras empresas do setor, bem como apresentar para as empresas estudadas possibilidade de aprimoramento de seu processo. No contexto acadêmico e para a comunidade científica, este estudo vem a contribuir para a investigação acerca da relação entre a abrangência dos níveis de gaps de competência e as abordagens reativa e proativa do processo de avaliação da necessidade de treinamento ambiental, aprofundando a investigação sobre os temas.

As limitações desta pesquisa pautam-se, essencialmente, na própria estratégia de pesquisa, que não poderia ser levada à generalização, bem como na necessidade de aprofundamento do próprio estudo envolvendo outras áreas organizacionais ou a ampliação para investigação de unidades das empresas situadas fora do Brasil, o que abre possibilidade para continuidade do estudo. As contribuições apontadas, assim como a abertura de possibilidade para continuidade, consequentemente, encarregam-se da criação de uma agenda de pesquisas que possa propiciar a formação do conhecimento sobre o tema.

\section{REFERÊNCIAS}

Abbad, G. D. S., \& Mourão, L. (2012). Avaliação de necessidades de TD\&E: proposição de um novo modelo. Revista de Administração Mackenzie, 13(6), 107.

ABIQUIM - Associação Brasileira das Indústrias Químicas (2017). Site disponível em http://abiquim.org.br. Acesso em 23/01/2017.

Aguinis, H \& Kraiger, K. (2009). Benefits of training and development for individuals and teams, organizations, and society. Annual Review of Psychology, 60, 451-474.

Arshad, M. A., Yusof, A. N. M., Mahamood, A., Ahmed, A. \& Akhtar, S. (2015). A Study on Training Needs Analysis (TNA) Process among Manufacturing Companies Registered with Pembangunan Sumber Manusia Berhad (PSMB) at Bayan Lepas Area, Penang, Malaysia. Mediterranean Journal of Social Sciences, 6(4), 670.

Baccaro, T. A., Caldana, A. C. F., \& Shinyashiki, G. T. (2015). Influência do Treinamento Ambiental na Consciência Ambiental de Profissionais da Área de Recursos Humanos. REGE Revista de Gestão, 22(2), 241-255.

Cherian, J., \& Jacob, J. (2012). A study of green HR practices and its effective implementation in the organization: a review. International journal of business and Management, 7(21), 25.

Collins, E., Lawrence, S., Pavlovich, K. \& Ryan, C. (2007). Business networks and the uptake of sustainability practices: the case of New Zealand. Journal of Cleaner Production 15, 729-740.

Daily, B. F., \& Huang, S. C. (2001). Achieving sustainability through attention to human resource factors in environmental management. International Journal of Operations \& Production Management, 21(12), 1539-1552. 
Daily, B. F., Bishop, J. W. \& Massoud, J. A. (2012). The role of training and empowerment in environmental performance: A study of the Mexican maquiladora industry. International Journal of Operations \& Production Management, 32(5), 631-647.

Dermol, V., Cater, T. (2013). The influence of training and training transfer factors on organisational learning and performance. Personnel review, 42(3), 324-348.

Ferreira, R. R. \& Abbad, G. (2013). Training needs assessment: where we are and where we should go. BAR-Brazilian Administration Review, 10(1), 77-99.

Ferreira, V. F., Da Rocha, D. R. \& Da Silva, F. C. (2013). Química Verde, Economia Sustentável e Qualidade de Vida. Revista Virtual de Química, 6(1), 85-111.

Gil, A. C. (2009). Estudo de Caso: Fundamentação científica subsídios para coleta e análise de dados como redigir o relatório. São Paulo: Atlas.

Godoy, A. S. (2006). Estudo de caso qualitativo. In: Godoi, C. K., Bandeira-De-Melo, R. \& Silva, A. B. (Orgs.) Pesquisa qualitativa em estudos organizacionais paradigmas, estratégias e métodos. São Paulo: Saraiva, p. 115-146.

ISO:14001. (2015). Sistemas de Gestão Ambiental-Especificação e Diretrizes para Uso. ABNT-Associação Brasileira de Normas Técnicas, Rio de Janeiro, RJ.

Jabbour, C. J. C. (2015). Environmental training and environmental management maturity of Brazilian companies with ISO14001: empirical evidence. Journal of Cleaner Production, 96, 331-338.

Jabbour, C. J. C. (2013). Environmental training in organizations: From a literature review to a framework for future research. Resources, Conservation and Recycling, 74,144-155.

Jabbour, C. J. C. (2011). How green are HRM practices, organizational culture, learning and teamwork? A Brazilian study. Industrial and Commercial Training, 43(2), 98-105.

Jabbour, C. J. C. \& Santos, F. C. A. (2008). Relationships between human resource dimensions and environmental management in companies: proposal of a model. Journal of Cleaner Production, 16(1), 51-58.

Leite, N. R. P.; et al. (2009). Treinamento, Desenvolvimento e Educação de Pessoas: um desafio na estratégia organizacional. In: Albuquerque, L. G.; Leite, N. P. (Orgs). Gestão de Pessoas: perspectivas estratégicas. p. 3-16, São Paulo: Atlas.

Mason, J. (1996). Planning and designing qualitative research. In: Mason, J. Qualitative Researching. London: Sage, p. 9-19.

Moraes, S. S. \& Battistelle, R. A. G. (2015). Treinamento ambiental: revisão de literatura e sugestões de pesquisa. Gepros: Gestão da Produção, Operações e Sistemas, 10(2), 115.

Narasimhan, G. V. \& Ramanarayanan, C. S. (2014). Analysis of Training Needs Assessment and Implementation - A Comparative Study of Public and Private Sector Banks. Indian Journal of Commerce and Management Studies, $5(3), 71$.

Prado, A. G. S. (2003). Química verde, os desafios da química do novo milênio. Química Nova, 26(5), 738-744.

Ramus, C. A. (2002). Encouraging innovative environmental actions: what companies and managers must do. Journal of world business, 37(2), 151-164.

Reed, J. \& Vakola, M. (2006). What role can a training needs analysis play in organisational change?. Journal of Organizational Change Management, 19(3), 393-407. 
Salas, E. \& Cannon-Bowers, J. A. (2001). The science of training: A decade of progress. Annual review of psychology, 52(1), 471-499.

Soto, J. (2012). A química sustentável: desafios, dilemas e perspectivas. Desenvolvimento sustentável 2012-2050: visão, rumos e contradições. Almeida, F. (Org). Rio de Janeiro, Elsevier.

Teixeira, A. A., Jabbour, C. J. C., de Sousa Jabbour, A. B. L., Latan, H., \& de Oliveira, J. H. C. (2016). Green Training and Green Supply Chain Management: Evidence from Brazilian Firms. Journal of Cleaner Production, 116, $170-$ 176.

Unnikrishnan, S. \& Hegde, D. S. (2007). Environmental training and cleaner production in Indian industry - A micro-level study. Resources, Conservation and Recycling, 50(4), 427-441.

Yin, R. (2010). Estudo de Caso: Planejamento e Métodos (4a. ed.). Porto Alegre: Bookman. 